\title{
Preliminary Results of DHEA in Poor Responders in IVF
}

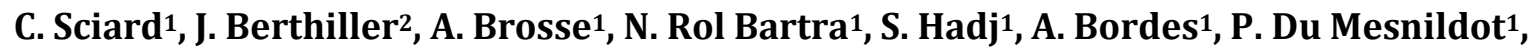 \\ J. Lornage ${ }^{3}$, H. Lejeune1, I. Plotton ${ }^{1,4}$, B. Salle1

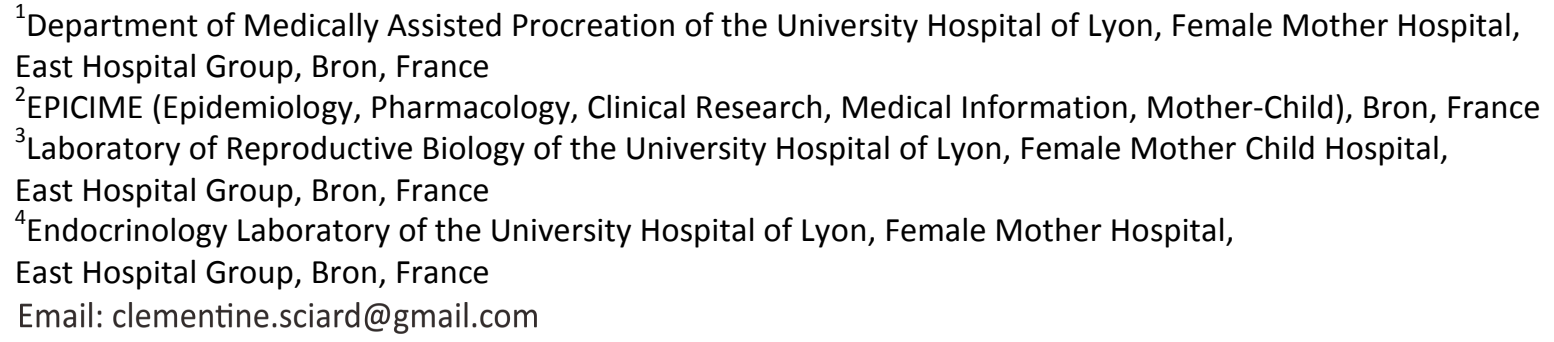

Received 23 February 2016; accepted 4 June 2016; published 8 June 2016

Copyright (C) 2016 by authors and Scientific Research Publishing Inc.

This work is licensed under the Creative Commons Attribution International License (CC BY).

http://creativecommons.org/licenses/by/4.0/

(c) (i) Open Access

\begin{abstract}
Objective: Dehydroepiandrosterone (DHEA) has been previously reported to improve ovarian response in IVF for poor responders. Its effect remains uncertain. The purpose of this study was to evaluate the effect of DHEA given during two years in our unit, in order to build hypothesis for a future randomized controlled trial. Design: Cohort of exposed and non-exposed DHEA study. Patient(s): 224 patients with AMH under $1.6 \mathrm{ng} / \mathrm{ml}$ undergoing IVF with a short antagonist protocol adding FSH and LH rec were enrolled for 327 IVF cycles. 176 patients had been treated with DHEA before IVF, and 151 had not. Intervention(s): None. Main Outcome Measure(s): Cancellation cycle rate, amount of gonadotrophins used, estradiol level on day six of stimulation, number of oocytes retrieved, metaphase II oocytes, blastocysts and frozen embryos, clinical pregnancy rate and miscarriage rate. Results: The cancellation rate was significantly higher in the group without DHEA $(29.8 \%$ versus $13.1 \%, p=0.002)$. The amount of gonadotrophins used for stimulation, the estradiol level on day six of stimulation, the number of oocytes retrieved, metaphase II oocytes, blastocysts, frozen embryos and miscarriage rate were not different in both groups. The clinical pregnancy rate per included patient in the DHEA group was $17.1 \%$ versus $8.6 \%$ in the non-treated group ( $p=0.02)$. Conclusion: DHEA appears to improve clinical pregnancy rate by decreasing the cancellation rate.
\end{abstract}

\section{Keywords}

DHEA, IVF, Poor Responders

How to cite this paper: Sciard, C., Berthiller, J., Brosse, A., Bartra, N.R., Hadj, S., Bordes, A., Du Mesnildot, P., Lornage, J., Lejeune, H., Plotton, I. and Salle, B. (2016) Preliminary Results of DHEA in Poor Responders in IVF. Open Journal of Obstetrics and Gynecology, 6, 396-403. http://dx.doi.org/10.4236/ojog.2016.67052 


\section{Introduction}

Poor ovarian response (POR) represents a significant challenge in assisted reproduction. It is encountered in 5\% to $18 \%$ of women undergoing in vitro fertilization (IVF) [1] [2]. It is increasing because of the delay to the first pregnancy: about 26.5 years old in 1977, between 29 and 31 in 2009 in most of European countries [3]. Patient with POR have very low successful pregnancy rate: $2 \%$ to $18 \%$, depending on maternal age and number of oocytes retrieved [4]. Less oocytes are collected, and lower is the pregnancy rate.

Over the years, many adjuvant therapies or protocols have been tested for POR to improve clinical pregnancy rate such as gonadotrophins-releasing hormone $(\mathrm{GnRH})$ flare protocols, high dose of gonadotrophins, antagonist protocol, arginine, testosterone, letrozole, luteal estradiol, or low dose of aspirin [5]-[14]. A recent study showed that addition of r-LH to r-FSH increased pregnancy rate in POR patients [15]. The Cochrane review has concluded that adjuvant therapy offered no clear benefit and could not be recommended [16].

Idea that dehydroepiandrosterone (DHEA) could be an adjuvant therapy appeared in 2000 with Casson et al. [17]. DHEA is an endogenous steroid synthesized by the adrenocortical reticularis zone and ovarian theca cells [18]. It is converted into androstenedione and estrone, the source of testosterone and estradiol according to the two cell theory [19] [20]. This hormone circulates in high amount before menopause with a peak at the age of 20 years and decreases progressively with age [21].

Its mechanism of action on the ovary remains hypothetic. Different hypothesis have been described over the years. It may increase the bioavailability of IGF-I, as reflected by an increase in IGF-I and a decrease in IGFBP-1 levels [17] [22]. IGF1 may potentiate gonadotrophins actions [17]. Gleicher et al. reported that DHEA created a polycystic ovary (PCO) like characteristics during stimulation [23]. It had indeed been used to induce PCO like ovarian phenotypes in rats and mice [24] [25]. An increased level of androgens characterizes this PCO situation. And androgens had previously been reported to suppress apoptosis [26] [27]. Hyman et al. suggests that DHEA does not influence early state of folliculogenesis but antral follicles representing the recruitable follicles cohort that has escaped from atresia [28].

Effect of DHEA is controversial. Many studies showed a significant clinical pregnancy rate improvement [2] [17] [28]-[30]. Other authors did not found statistically significant difference in the outcome of IVF treatment [3] [31]-[33]. Most of the studies are retrospectives and observational or small effectives randomized controlled trials. Despite insufficient evidence, there is actually a worldwide use of DHEA, which is given to $25.8 \%$ of the POR patients [34]. It seems important to evaluate this self-service treatment suitability.

We did a pilot study in our hospital in Lyon to make a current situation of our practices regarding DHEA. This study can help us to build hypothesis for our future randomized, controlled, multi-center, large population trial on DHEA versus placebo we plan to organize.

This retrospective cohort study aims to compare IVF outcome in DHEA treated women versus no treatment.

\section{Materials and Methods}

\subsection{Patients and Design}

A cohort of exposed and non-exposed DHEA patient was performed in the Femme Mere Enfant hospital in Lyon, between January 2013 and December 2014. All patients with AMH under $1.6 \mathrm{ng} / \mathrm{ml}$ treated for an IVF cycle with an antagonist protocol adding FSH and LH were enrolled for this study. Women above 43 years old were not included. Patients were not excluded according to the Body Mass Index (BMI), the rank of stimulation or the parity. In our department, some patients with a predictable poor ovarian response had received a daily dose of $75 \mathrm{mg}$ DHEA for a minimum period of 60 days. The remaining patients with the same characteristics who did not receive DHEA were used as the control group. For the first part of the statistical analysis, all patients were included. Patients who had a cycle cancellation were excluded for the second part of the analysis.

\subsection{Treatment Protocols}

All patients underwent IVF cycle with the use of Gonadotrophins Releasing Hormone (GnRH) antagonist protocol adding FSH and LH. They began an oral combined hormonal contraceptive on the first period day. After 3 or 4 weeks of daily treatment, they stopped it and began stimulation five days later with 150 - 300 IU of recombinant FSH and $75-225$ of recombinant LH.

Response to ovarian stimulation was monitored by hormonal blood test on day 6 and we performed an ultra- 
sound every two or three day from day 6. Daily injection of GnRH antagonist was administered from the first control day to prevent premature ovulation. Recombinant hCG was given to trigger ovulation when at least three leading follicles reached $17 \mathrm{~mm}$. It was followed 36 hours later by transvaginal oocytes retrieval. ICSI was performed for all, as routine practice for PORs in our department. Number of oocytes, metaphase II oocytes, fertilized oocytes, cleavage embryos, blastocysts, number and day of embryos transferred or frozen, estradiol level on day 6 of stimulation, total dose of gonadotrophins were documented. Luteal phase was supported by vaginal progesterone $600 \mathrm{mg}$ a day from the day of retrieval. A quantitative pregnancy test, serum B HCG was performed 16 days after the day of retrieval. In case of pregnancy, an ultrasound was performed one month after the embryo transfer.

The stimulation was cancelled if less than three leading follicles reached $17 \mathrm{~mm}$ or when endometrium measured less than $6 \mathrm{~mm}$.

\subsection{Statistical Analysis}

Quantitative variables were expressed as means \pm standard deviation when the distribution was normal or median and minimum and maximum when the distribution was not normal.

The hypothesis of normal distribution of quantitative variables was tested using the Kolmogorov-Smirnov and graphically confirmed with a histogram. If necessary, the variable will be converted to Log and/or specifically analyzed after exclusion of outliers.

Categorical variables were compared using the Chi 2 test or Fisher's exact test when the conditions of application of Chi square test were not met.

Quantitative variables were compared between groups using Student's t test after verification of equality of variances when data were normally distributed, and with the nonparametric test of Wilcoxon when the hypothesis of normality of distribution was not verified.

A logistic regression was conducted in order to quantify the association between patients' success to pregnancy or positive retrieval oocytes and explicative variable such as DHEA status, or AMH level, first as a univariate analysis and second as a multivariate analysis including significant variable in the univariate analysis and potential confounding factors such as age.

The statistical tests are bilateral and the level of significance was set to $5 \%(\mathrm{p}<0.05)$.

Statistical analyses were conducted using SAS version 9.1.3 (SAS Institute Inc, NC).

\section{Results}

During the study period, 224 patients were enrolled for 327 IVF cycles. 176 had been treated with DHEA before IVF, 151 had not. In the group with DHEA 153 had oocyte retrieval and 127 had embryo transfer versus 106 and 86 in the control group.

The demographics of the two groups are shown in Table 1. Patients treated with DHEA were significantly younger (35.9 versus 37.2 years old; $p=0.002)$, with a shorter infertility history (5.0 versus 5.9 years; $p=$ $0.002)$ and had more $\mathrm{AMH}<$ lower than $1.1 \mathrm{ng} / \mathrm{ml}(129$ versus $92 ; \mathrm{p}=0.01)$. There was no difference between the two groups regarding the type of infertility, the AMP rank, AFC or spermatic pathology.

The cancellation rates were significantly higher in the group without DHEA $(29.8 \%$ versus $13.1 \%, p=0.002)$ (Table 1). Among retrieved patients, four (0.03\%) had no oocyte and $22(14.4 \%)$ had no embryo transferred in the DHEA group versus one $(0.001 \%)$ and $19(18.0 \%)$ in the DHEA treated group, which was not different between both groups.

The amount of gonadotrophins used for stimulation and the estradiol level on day six of stimulation was not different in both groups. There was no improvement in the number of oocytes retrieved, metaphase II oocytes, blastocysts or frozen embryos (Table 2).

The clinical pregnancy rate per included patient in the DHEA group was $17.1 \%$ versus $8.6 \%$ in the nontreated group $(\mathrm{p}=0.02)$ (Table 3$)$. This difference was significant independently from the age and the AMH level $(\mathrm{p}=0.0002$, data not shown). Due to sparse data and small sample size, additional adjustment was not conducted.

The clinical pregnancy rate per retrieval in the DHEA group was $23.6 \%$ versus $15.1 \%$ in the non-treated group $(\mathrm{p}=0.13)$ (Table 3$)$.

The clinical pregnancy rate per transfer in the DHEA group was $17.3 \%$ versus $9.3 \%$ in the non-treated group $(\mathrm{p}=0.1)$ (Table 3). 
Table 1. Characteristic of patients (327 IVF cycles).

\begin{tabular}{cccc}
\hline & No DHEA & DHEA value \\
age & 151 & 176 & $0.002 /$ \\
infertility duration & $37.2(4.0)$ & $35.9(4.3)$ & $0.002 /$ \\
primary infertility & $5.9(2.5)$ & $5(2.5)$ & $0.5 \#$ \\
secondary infertility & $72(52.5)$ & $109(65.6)$ & $34(21.7)$ \\
primo-secondary infertility & $46(33.6)$ & $17(10.8)$ & $0.08 /$ \\
AMP rank & $13(9.5)$ & $2.1(1.3)$ & $0.01 \#$ \\
AMH $>1.1$ & $2.1(1.3)$ & $47(26.7)$ & $0.90 /$ \\
AFC & $59(39.1)$ & $6,9(3.6)$ & $0.88 \#$ \\
oligospermia & $7.2(4.6)$ & $41(28.1)$ & $0.48 \#$ \\
cryptozoospermia & $29(29.0)$ & $7(4.8)$ & $0.21 \#$ \\
sperm anomaly & $3(3.0)$ & $79(54.1)$ & $0.0002 \#$ \\
cancellation cycle & $54(54.0)$ & $23(13.1)$ & \\
\hline
\end{tabular}

\#Chi-square; /Wilcoxon.

Table 2. Stimulation characteristics, biological and clinical evolution in patients without cancellation.

\begin{tabular}{cccc}
\hline & No DHEA & DHEA & p value \\
gonadotrophin & 86 & 127 & \\
dose (IU) & & & $0.97 /$ \\
duration (d) & $4231.9(905.3)$ & $4238.4(885.8)$ & $0.58 /$ \\
estradiol level on day 6 & $11.36(1.8)$ & $11.49(1.7)$ & $0.33 /$ \\
number & $326.27(300.3)$ & $344.69(252.0)$ & $0.73 /$ \\
oocyte retrived & & $6.31(3.9)$ & $0.16 /$ \\
metaphase 2 oocytes & $6.21(4.0)$ & $5.75(3.8)$ & $0.18 /$ \\
blastocysts & $5.05(3.5)$ & $0.98(1.8)$ & $0.99 /$ \\
\hline
\end{tabular}

/Wilcoxon.

Table 3. Clinical pregnancy rate.

\begin{tabular}{cccc}
\hline & No DHEA & DHEA & p value \\
\hline per included patient $(\mathrm{n}=326)$ & $13(8.6 \%)$ & $30(17.1 \%)$ & $0.02 \#$ \\
per retrieval $(\mathrm{n}=213)$ & $13(15.1 \%)$ & $30(23.6 \%)$ & $0.13 \#$ \\
per transfer $(\mathrm{n}=213)$ & $8(9.3 \%)$ & $22(17.3 \%)$ & $0.10 \#$ \\
\hline
\end{tabular}

\footnotetext{
${ }^{\#}$ Chi-square.
} 
Among patients transferred, the miscarriage rate was $4.6 \%$ in the non-treated group and $6.3 \%$ in the DHEA group $(\mathrm{p}=0.6)$.

\section{Discussion}

The critical evaluation of the many published protocols on DHEA and fertility is very difficult mainly because of a lack of uniformity in the definition of "poor responders. To avoid this, Ferraretti et al. on behalf of the ESHRE, presented Bologna's criteria to standardize the definition of POR [35]. Only few studies have used it since [29] [32]. In other studies, poor responders are defined according to the number of oocytes retrieved, ultrasound findings, use of hormonal ovarian reserve markers such as day 3 FSH or AMH. For this study, we did not use the Bologna's criteria either: the practice in our department is to enlarge our treated population to patients with an AMH level less than $1.6 \mathrm{ng} / \mathrm{ml}$. We noted that our population treated with DHEA is significantly younger than the non-treated group, which is why we did a multivariate analysis and concluded that the differences observed are independent from the age. It seems that we have been giving DHEA to the patients with a significantly lower AMH and the worst prognosis. We can also criticize the fact that AMH has been tested in different laboratories with different kits. However, the variability between methods is weak for the lower values so we can consider it rather reliable. This marker is also used on a daily basis in our ward.

Our patients were going through a short antagonist protocol adding r-FSH and r-LH. A significant benefit of r-FSH plus r-LH versus r-FSH alone on clinical pregnancy rate in the poor responders subgroup (RR $1.30 ; 95 \%$ CI 1.01 - 1.67) was demonstrated in a meta-analysis done by Lehert and al [15]. Sunkara et al. showed that there was no significant difference between long agonist and antagonist regimens (4.42 \pm 3.06 vs. $3.30 \pm 2.91)$ [36]. In our unit we are used to give a short antagonist to POR patients and most of the recent studies on DHEA also used this protocol [29] [31] [32].

As noted previously we found that cancellation rate caused by poor response were significantly lower in the DHEA group. In Barad et al. study, the cycle cancellation rate was 32\% without DHEA versus $4 \%$ post treatment $(p=0.02)$ [23]. Poli et al. also showed a significant reduction of cancelled cycles $(p=0.03)[37]$. In contrast, cancellation rate were not different between the two groups in the prospective case-control study made by Jirge et al. but only 31 patients were included [29].

Our study did not find any improvement in any of the stimulation characteristics (gonadotrophins requirements, duration of stimulation oocytes and day 6 estradiol). Other authors have previously reported an improvement. Hyman et al. showed an increased peak E2 levels on HCG day (from $2607 \pm 2143$ to $4710 \pm 3869$ $\mathrm{pmol} / \mathrm{L} \mathrm{p}=0.0005$ ) [28]. Singh et al. also reported a significant increase in the serum AMH, peak E2 level on HCG day [38]. In our unit, blood sample are obtained only on day 6 of stimulation, but is not done later during the stimulation and specifically not on HCG day since we used an antagonist: this can explain the difference. In contrast and as noted in our study, Vlahos et al. did not found any statistical difference in stimulation duration (from 9.17 to 9.20 days with DHEA) or total gonadotrophins requirement (from 3936 to 3742 UI with DHEA) in his study, which includes 48 POR according to Bologna's criteria and 113 controls [32].

Concerning the number of oocytes retrieved, we did not observe a significant difference between the two groups. In the last randomized controlled trial made by Kara et al, results were not different from ours in terms of number of oocytes retrieved: 5.74 (3.69) in the DHEA group versus 5.35 (3.45) in the control group ( $p=$ 0.44) [39]. As shown in Table 2, the number of oocytes retrieved in our study is not different. Whereas, Barad showed a significant increase of oocytes retrieved [23]. But it was a cross match study with only 25 patients. Poli et al. also showed a higher number of oocytes retrieved $(\mathrm{p}<0.01)$ but it included only 29 patients [37].

Our study showed a significant higher pregnancy rate per included patient in the group treated with DHEA. We observed a higher pregnancy rate per retrieval and transfer also it did not reach significance, maybe due to a too small sample size in these two groups. Results of the three different randomized controlled trials are controversial. Wiser et al. showed a significantly higher live birth rate among study group after two treatment cycles: $23.0 \%$ in the DHEA group versus $4.0 \%$ in the control group $(p=0.05)$ [2]. But it was found after pooling the results of two treatment cycles with a p-value of 0.05 . Yeung et al. showed no statically significant difference in the rate of clinical pregnancy $18.8 \%$ versus $25.0 \%(\mathrm{p}=0.38)$ but it included only 16 patients in each arms [31]. Kara et al. found 32 and $33 \%$ of pregnancy rate in both group but patients were very young (about 30 years old) and pregnancy rate is in astonishment extremely high for the POR population [39].

Narkwichean et al. demonstrated in their meta-analysis a trend of a positive effect of DHEA on pregnancy 
rate despite a lack of significance. It was marginally superior in the DHEA group (RR 1.87) [3]. Li et al. also made a meta-analysis and found an improvement in the pregnancy rate in the DHEA group (RR 2, p = 0.02) [40].

DHEA has previously been reported to decrease miscarriage rate. Gleicher et al. showed that after two months of DHEA supplementation, miscarriage rate was significantly lower, most pronounced above 35years [41]. They then found out that it was due to a significant decrease of number and percentage of embryos with aneuploidy [42]. In the present study, the miscarriage rate is not different between both groups, $5.0 \%$ in each but the effective is very low (five and seven miscarriages in each group).

None of the studies on DHEA reported major side effects. Only minor masculinity side effects have been observed such as oily skin, transitional acne, hair loss [17] [30] [31] [41] [43] [44]. Improved feeling of well being and increased libido while using DHEA has been reported [23]. Our study did not reveal any major side effect either.

The aim of this study was to have a feedback of the practice in our ward regarding DHEA, to confirm the interest of a larger study and to build hypothesis for a future randomized controlled trial in our unit and to have a quick feedback of DHEA in our population. It explains why it is not randomized and suffers from all inherent problems of this kind of studies. To limit the weakness of our study we included a high number of patients, which is one of the major strength of our study. To the best of our knowledge this is one of the first studies including such a large cohort of patients (327 stimulation cycles on 224 patients). We collected data in a prospective way so there is no recall bias and we have a low rate of missing data. We accepted the different bias since it was a preliminary study before a multicenter randomized control trial.

\section{Conclusion}

A significant decrease in cancellation rate was observed in poor responders who received DHEA. This group had significant higher pregnancy rate per included patient but the difference was not significant regarding the pregnancy rate per retrieval or per transfer. A further RCT should be done soon in our unit to evaluate the effect of DHEA with a better reliability.

\section{References}

[1] Pellicer, A., Lightman, A., Diamond, M.P., Russell, J.B. and De Cherney, A.H. (1987) Outcome of in Vitro Fertilization in Women with Low Response to Ovarian Stimulation. Fertility and Sterility, 47, 812-815. http://dx.doi.org/10.1016/S0015-0282(16)59170-5

[2] Wiser, A., Gonen, O., Ghetler, Y., Shavit, T., Berkovitz, A. and Shulman, A. (2010) Addition of Dehydroepiandrosterone (DHEA) for Poor-Responder Patients before and during IVF Treatment Improves the Pregnancy Rate: A Randomized Prospective Study. Human Reproduction (Oxford, England), 25, 2496-500. http://dx.doi.org/10.1093/humrep/deq220

[3] Narkwichean, A., Maalouf, W., Campbell, B.K. and Jayaprakasan, K. (2013) Efficacy of Dehydroepiandrosterone to Improve Ovarian Response in Women with Diminished Ovarian Reserve: A Meta-Analysis. Reproductive Biology and Endocrinology: $R B$ \& E, 11, 44. http://dx.doi.org/10.1186/1477-7827-11-44

[4] Ulug, U., Ben-Shlomo, I., Turan, E., Erden, H.F., Akman, M.A. and Bahceci, M. (2003) Conception Rates Following Assisted Reproduction in Poor Responder Patients: A Retrospective Study in 300 Consecutive Cycles. Reproductive Biomedicine Online, 6, 439-443. http://dx.doi.org/10.1016/S1472-6483(10)62164-5

[5] Hofmann, G.E., Toner, J.P., Muasher, S.J. and Jones, G.S. (1989) High-Dose Follicle-Stimulating Hormone (FSH) Ovarian Stimulation in Low-Responder Patients for in Vitro Fertilization. Journal of in Vitro Fertilization and Embryo Transfer: IVF, 6, 285-289. http://dx.doi.org/10.1007/BF01139183

[6] Padilla, S.L., Dugan, K., Maruschak, V., Shalika, S. and Smith, R.D. (1996) Use of the Flare-Up Protocol with High Dose Human Follicle Stimulating Hormone and Human Menopausal Gonadotropins for in Vitro Fertilization in Poor Responders. Fertility and Sterility, 65, 796-799. http://dx.doi.org/10.1016/S0015-0282(16)58216-8

[7] Hugues, J.N. and Cédrin Durnerin, I.C. (1998) Revisiting Gonadotrophin-Releasing Hormone Agonist Protocols and Management of Poor Ovarian Responses to Gonadotrophins. Human Reproduction Update, 4, 83-101. http://dx.doi.org/10.1093/humupd/4.1.83

[8] Surrey, E.S., Bower, J., Hill, D.M., Ramsey, J. and Surrey, M.W. (1998) Clinical and Endocrine Effects of a Microdose GnRH Agonist Flare Regimen Administered to Poor Responders who Are Undergoing in Vitro Fertilization. Fertility and Sterility, 69, 419-424. http://dx.doi.org/10.1016/s0015-0282(97)00575-x 
[9] Battaglia, C., Salvatori, M., Maxia, N., Petraglia, F., Facchinetti, F. and Volpe, A. (1999) Adjuvant L-Arginine Treatment for in Vitro Fertilization in Poor Responder Patients. Human Reproduction (Oxford, England), 14, 1690-1697. http://dx.doi.org/10.1093/humrep/14.7.1690

[10] Franco, J.G., Baruffi, R.L.R., Mauri, A.L., Petersen, C.G., Felipe, V., Cornicelli, J., et al. (2006) GnRH Agonist versus GnRH Antagonist in Poor Ovarian Responders: A Meta-Analysis. Reproductive Biomedicine Online, 13, 618-627. http://dx.doi.org/10.1016/S1472-6483(10)60651-7

[11] Frattarelli, J.L., McWilliams, G.D.E., Hill, M.J., Miller, K.A. and Scott, R.T. (2008) Low-Dose Aspirin Use Does Not Improve in Vitro Fertilization Outcomes in Poor Responders. Fertility and Sterility, 89, 1113-1117. http://dx.doi.org/10.1016/j.fertnstert.2007.05.007

[12] Garcia-Velasco, J.A., Moreno, L., Pacheco, A., Guillén, A., Duque, L., Requena, A., et al. (2005) The Aromatase Inhibitor Letrozole Increases the Concentration of Intraovarian Androgens and Improves in Vitro Fertilization Outcome in Low Responder Patients: A Pilot Study. Fertility and Sterility, 84, 82-87. http://dx.doi.org/10.1016/j.fertnstert.2005.01.117

[13] Hill, M.J., McWilliams, G.D.E., Miller, K.A., Scott, R.T. and Frattarelli, J.L. (2009) A Luteal Estradiol Protocol for Anticipated Poor-Responder Patients May Improve Delivery Rates. Fertility and Sterility, 91, 739-743. http://dx.doi.org/10.1016/j.fertnstert.2007.12.073

[14] Schoolcraft, W.B., Surrey, E.S., Minjarez, D.A., Stevens, J.M. and Gardner, D.K. (2008) Management of Poor Responders: Can Outcomes Be Improved with a Novel Gonadotropin-Releasing Hormone Antagonist/Letrozole Protocol? Fertility and Sterility, 89, 151-156. http://dx.doi.org/10.1016/j.fertnstert.2007.02.013

[15] Lehert, P., Kolibianakis, E.M., Venetis, C.A., Schertz, J., Saunders, H., Arriagada, P., et al. (2014) Recombinant Human Follicle-Stimulating Hormone (r-hFSH) Plus Recombinant Luteinizing Hormone versus r-hFSH Alone for Ovarian Stimulation during Assisted Reproductive Technology: Systematic Review and Meta-Analysis. Reproductive Biology and Endocrinology: $R B \&$ E, 12, 17. http://dx.doi.org/10.1186/1477-7827-12-17

[16] Pandian, Z., McTavish, A.R., Aucott, L., Hamilton, M.P. and Bhattacharya, S. (2010) Interventions for "Poor Responders" to Controlled Ovarian Hyper Stimulation (COH) in in Vitro Fertilization (IVF). Cochrane Database of Systematic Reviews, No. 1, Article No. CD004379. http://dx.doi.org/10.1002/14651858.cd004379.pub3

[17] Casson, P.R., Lindsay, M.S., Pisarska, M.D., Carson, S.A. and Buster, J.E. (2000) Dehydroepiandrosterone Supplementation Augments Ovarian Stimulation in Poor Responders: A Case Series. Human Reproduction (Oxford, England), 15, 2129-2132. http://dx.doi.org/10.1093/humrep/15.10.2129

[18] Burger, H.G. (2002) Androgen Production in Women. Fertility and Sterility, 77, S3-S55. http://dx.doi.org/10.1016/S0015-0282(02)02985-0

[19] Ben-Chetrit, A., Gotlieb, L., Wong, P.Y. and Casper, R.F. (1996) Ovarian Response to Recombinant Human Follicle-Stimulating Hormone in Luteinizing Hormone-Depleted Women: Examination of the Two Cell, Two Gonadotropin Theory. Fertility and Sterility, 65, 711-717. http://dx.doi.org/10.1016/S0015-0282(16)58201-6

[20] McNatty, K.P., Makris, A., Reinhold, V.N., De Grazia, C., Osathanondh, R. and Ryan, K.J. (1979) Metabolism of Androstenedione by Human Ovarian Tissues in Vitro with Particular Reference to Reductase and Aromatase Activity. Steroids, 34, 429-443. http://dx.doi.org/10.1016/0039-128X(79)90104-1

[21] Orentreich, N., Brind, J.L., Rizer, R.L. and Vogelman, J.H. (1984) Age Changes and Sex Differences in Serum Dehydroepiandrosterone Sulfate Concentrations throughout Adulthood. The Journal of Clinical Endocrinology and Metabolism, 59, 551-555. http://dx.doi.org/10.1210/jcem-59-3-551

[22] Morales, A.J., Nolan, J.J., Nelson, J.C. and Yen, S.S. (1994) Effects of Replacement Dose of Dehydroepiandrosterone in Men and Women of Advancing Age. The Journal of Clinical Endocrinology and Metabolism, 78, 1360-1367. http://dx.doi.org/10.1210/jcem.78.6.7515387

[23] Barad, D. and Gleicher, N. (2006) Effect of Dehydroepiandrosterone on Oocyte and Embryo Yields, Embryo Grade and Cell Number in IVF. Human Reproduction (Oxford, England), 21, 2845-2849. http://dx.doi.org/10.1093/humrep/del254

[24] Mahajan, D.K. (1988) Polycystic Ovarian Disease: Animal Models. Endocrinology and Metabolism Clinics of North America, 17, 705-732.

[25] Luchetti, C.G., Solano, M.E., Sander, V., Arcos, M.L.B., Gonzalez, C., Di Girolamo, G., et al. (2004) Effects of Dehydroepiandrosterone on Ovarian Cystogenesis and Immune Function. Journal of Reproductive Immunology, 64, 59-74. http://dx.doi.org/10.1016/j.jri.2004.04.002

[26] Billig, H., Furuta, I. and Hsueh, A.J. (1993) Estrogens Inhibit and Androgens Enhance Ovarian Granulosa Cell Apoptosis. Endocrinology, 133, 2204-2212.

[27] Kaipia, A. and Hsueh, A.J. (1997) Regulation of Ovarian Follicle Atresia. Annual Review of Physiology, 59, $349-363$. http://dx.doi.org/10.1146/annurev.physiol.59.1.349 
[28] Hyman, J.H., Margalioth, E.J., Rabinowitz, R., Tsafrir, A., Gal, M., Alerhand, S., et al. (2013) DHEA Supplementation May Improve IVF Outcome in Poor Responders: A Proposed Mechanism. European Journal of Obstetrics, Gynecology and Reproductive Biology, 168, 49-53. http://dx.doi.org/10.1016/j.ejogrb.2012.12.017

[29] Jirge, P.R., Chougule, S.M., Gavali, V.G. and Bhomkar, D.A. (2014) Impact of Dehydroepiandrosterone on Clinical Outcome in Poor Responders: A Pilot Study in Women Undergoing in Vitro Fertilization, Using Bologna Criteria. Journal of Human Reproductive Sciences, 7, 175-180. http://dx.doi.org/10.4103/0974-1208.142477

[30] Barad, D., Brill, H. and Gleicher, N. (2007) Update on the Use of Dehydroepiandrosterone Supplementation among Women with Diminished Ovarian Function. Journal of Assisted Reproduction and Genetics, 24, 629-634. http://dx.doi.org/10.1007/s10815-007-9178-X

[31] Yeung, T.W.Y., Chai, J., Li, R.H.W., Lee, V.C.Y., Ho, P.C. and Ng, E.H.Y. (2014) A Randomized, Controlled, Pilot Trial on the Effect of Dehydroepiandrosterone on Ovarian Response Markers, Ovarian Response, and in Vitro Fertilization Outcomes in Poor Responders. Fertility and Sterility, 102, 108-115.e1. http://dx.doi.org/10.1016/j.fertnstert.2014.03.044

[32] Vlahos, N., Papalouka, M., Triantafyllidou, O., Vlachos, A., Vakas, P., Grimbizis, G., et al. (2015) Dehydroepiandrosterone Administration before IVF in Poor Responders: A Prospective Cohort Study. Reproductive Biomedicine Online, 30, 191-196.

[33] Sunkara, S.K., Pundir, J. and Khalaf, Y. (2011) Effect of Androgen Supplementation or Modulation on Ovarian Stimulation Outcome in Poor Responders: A Meta-Analysis. Reproductive Biomedicine Online, 22, 545-555. http://dx.doi.org/10.1016/j.rbmo.2011.01.015

[34] Leong, M. and Patrizio, P. (2014) Poor Responders: How to Define, Diagnose and Treate?

[35] Ferraretti, A.P., La Marca, A., Fauser, B.C.J.M., Tarlatzis, B., Nargund, G., Gianaroli, L., et al. (2011) ESHRE Consensus on the Definition of "Poor Response" to Ovarian Stimulation for in Vitro Fertilization: The Bologna Criteria. Human Reproduction (Oxford, England), 26, 1616-1624. http://dx.doi.org/10.1093/humrep/der092

[36] Sunkara, S.K., Coomarasamy, A., Faris, R., Braude, P. and Khalaf, Y. (2014) Long Gonadotropin-Releasing Hormone Agonist versus Short Agonist versus Antagonist Regimens in Poor Responders Undergoing in Vitro Fertilization: A Randomized Controlled Trial. Fertility and Sterility, 101, 147-153. http://dx.doi.org/10.1016/j.fertnstert.2013.09.035

[37] Poli, E., Manfé, S., Capuzzo, D., Gava, S., Viganò, F., Coronella, M.L., et al. (2014) DHEA Pre-Treated Patients, Poor Responders to a First IVF (ICSI) Cycle: Clinical Results. Clinical and Experimental Obstetrics \& Gynecology, 41, 5-9.

[38] Singh, N., Zangmo, R., Kumar, S., Roy, K.K., Sharma, J.B., Malhotra, N., et al. (2013) A Prospective Study on Role of Dehydroepiandrosterone (DHEA) on Improving the Ovarian Reserve Markers in Infertile Patients with Poor Ovarian Reserve. Gynecological Endocrinology: The Official Journal of the International Society of Gynecological Endocrinology, 29, 989-992. http://dx.doi.org/10.3109/09513590.2013.824957

[39] Kara, M., Aydin, T., Aran, T., Turktekin, N. and Ozdemir, B. (2014) Does Dehydroepiandrosterone Supplementation really Affect IVF-ICSI Outcome in Women with Poor Ovarian Reserve? European Journal of Obstetrics, Gynecology and Reproductive Biology, 173, 63-65. http://dx.doi.org/10.1016/j.ejogrb.2013.11.008

[40] Li, J., Yuan, H., Chen, Y., Wu, H., Wu, H. and Li, L. (2015) A Meta-Analysis of dehydroepiandrosterone Supplementation among Women with Diminished Ovarian Reserve Undergoing in Vitro Fertilization or Intracytoplasmic Sperm Injection. International Journal of Gynecology \& Obstetrics, 131, 240-245.

[41] Gleicher, N., Ryan, E., Weghofer, A., Blanco-Mejia, S. and Barad, D.H. (2009) Miscarriage Rates after Dehydroepiandrosterone (DHEA) Supplementation in Women with Diminished Ovarian Reserve: A Case Control Study. Reproductive Biology and Endocrinology, 7, 108. http://dx.doi.org/10.1186/1477-7827-7-108

[42] Gleicher, N., Weghofer, A. and Barad, D.H. (2010) Dehydroepiandrosterone (DHEA) Reduces Embryo Aneuploidy: Direct Evidence from Preimplantation Genetic Screening (PGS). Reproductive Biology and Endocrinology, 8, 140. http://dx.doi.org/10.1186/1477-7827-8-140

[43] Kroboth, P.D., Salek, F.S., Pittenger, A.L., Fabian, T.J. and Frye, R.F. (1999) DHEA and DHEA-S: A Review. The Journal of Clinical Pharmacology, 39, 327-348. http://dx.doi.org/10.1177/00912709922007903

[44] Panjari, M., Bell, R.J., Jane, F., Adams, J., Morrow, C. and Davis, S.R. (2009) The Safety of 52 Weeks of Oral DHEA Therapy for Postmenopausal Women. Maturitas, 63, 240-245. http://dx.doi.org/10.1016/j.maturitas.2009.03.020 